\title{
Realistic 2D Facial Animation from One Image
}

\author{
Jaehwan Kim and Il-Kwon Jeong \\ Digital Content Research Division, ETRI, Korea \\ \{jh.kim,jik\}@etri.re.kr
}

\begin{abstract}
In this paper we present a novel complete framework for creating realistic facial animation given only one neutral facial image as an input data. Our approach is carried on in a two-dimensional image space, instead of threedimensional space. In addition, we employ an advanced computer vision method (digital image matting) as well as conventional image processing techniques (texture synthesis and image warping) in order to express more realistic facial animations. The major contribution of this work is showing how facial animation with a variety of realistic expressions can be generated very efficiently, where not only main facial components (e.g., eyeball, eyebrow and lip) but also pseudo-depth values obtained from their alpha mattes are utilized in our system. Simulations with real image confirm that our scheme produces high quality facial animations with an ease.
\end{abstract}

Keywords: two-dimensional facial animation, matting, warping, texture synthesis, realistic expression, pseudo-depth.

\section{Introduction}

Facial animation, the goal of which is to express the realistic appearance of a facial movement, plays an important role in a variety of applications including games, film productions, advertisements, educations and recently mobile contents. Hence, the creation of realistic animated faces is one of the most complicated and challenging tasks in computer graphics and computer vision areas. Conventional 3D physicsbased models in facial animations used computer graphics are based on a threedimensional anatomical model of the facial structure and a representation of its surface [1], [2]. These works would be definitely laborious and time-consuming. A major drawback of the three-dimensional facial animation approaches is its high computational cost of modeling and rendering. One of the earliest works on facial animation is done by Parke [3], [4], [5]. The Parke's parameterized facial model which can be generated as a mesh of three-dimensional points controlled by some parameters, is widely used in various fields including movie production studios so far because of its efficiency. Our proposed scheme is carried on in a two-dimensional image space without constructing three-dimensional facial reference model, under the Parke's assumption of facial expression animated by a set of parameters, so that the extensive computational cost problem can be overcome effectively. Moreover, our 
method is combined with existing some techniques (i.e., digital image matting [6], texture synthesis [7] and image warping [8]) dealing with extracting main facial components (e.g., eyeballs and lips) and restoring corrupted components for natural facial expressions. In this paper we introduce a novel complete framework for creating realistic facial animations given only one neutral facial $2 \mathrm{D}$ image as an input and show its high quality simulated results.

\section{Related Work}

For realism of the facial expressions, a detail like gaze direction is very important visual factor, but most of the image-based facial expressions [9], [10] just focus on subtle changes like facial wrinkles rather than an critical visualizations of facial components (e.g., eyeball and lip) movements. In the case of 3D physics-based facial animation [1], [2], 3D models of eyeballs must be designed manually and then would be utilized for eye movements as rotation of the models. In Bitouk et al. [11], they introduced a framework for creating a speech-enabled avatar from a single image by taking advantage of a 3D facial model for deformations of a prototype face. One of the main weak points of these works is that lots of manual efforts for $3 \mathrm{D}$ reference modeling are required, and which works would be definitely laborious. Hence, digital matting which is a kind of region segmentation method, has been widely used in image editing and film production application lately for providing an efficient way of tackling a complicated composition [6], [12], which makes it possible to extract facial components very accurately and realistically in our work.

\section{Realistic 2D Facial Animation from one Image}

Our system consists of three modules: facial feature points extraction, facial components extraction \& reconstruction, and facial expression parts.

\subsection{Normalization and Facial Features Extraction}

For 2D image-based facial animation system, it needs to extract the major feature points from an input face image firstly and then use it as a set of parameters for animating (i.e., image warping and components moving in here) facial movements. In this work, we semi-automatically extract the facial feature points from a neutral face image by incorporating SVM classifier [13] with our manual editing procedure.

Before extracting the major features, the face normalization should be performed by applying affine translation (1) based on user-defined points (e.g., both eyeballs locations) in order to define all feature points in a constant feature space:

$$
\hat{\boldsymbol{a}}=\left[\begin{array}{rr}
\cos \theta & -\sin \theta \\
\sin \theta & \cos \theta
\end{array}\right]\left[\begin{array}{cc}
s_{x} & 0 \\
0 & s_{y}
\end{array}\right] \boldsymbol{a}+\left[\begin{array}{l}
t_{x} \\
t_{y}
\end{array}\right],
$$




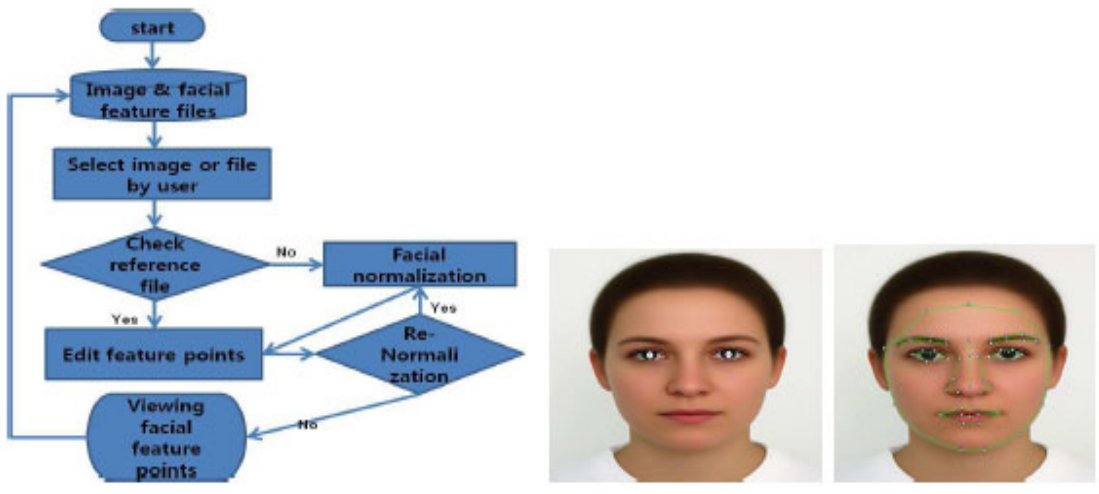

Fig. 1. Module of facial normalization \& feature extraction. Flow chart(left), raw input image and both eyeballs used for normalization(mid), normalized image \& defined 61 facial feature points(right)

where the variables $s_{x^{\prime}} \mathcal{S}_{y^{\prime}} t x^{c} t y$, and $\theta$ are for the horizontal and vertical scaling, translation and rotation, respectively. a and $\hat{a}$ are feature points of two static eyeballs in each raw and normalized face images. In here, we provide two modes depend on the count of feature points (i.e., 21 or 61 feature points mode can be selected by user). Meanwhile, both the computational cost and the animating realism according to the count of feature points are in a trade-off relationship.

\subsection{Facial Components Extraction and Reconstruction}

Once the defined feature points have been extracted semi-automatically, key facial components (e.g., lips and eyeballs) for making expressions are also segmented through a digital matting method [6]. The goal of digital matting is to estimate the opacity, the alpha matte, under an assumption that each pixel value is a linear combination of the corresponding foreground and background colors, which can be represented as following equation:

$$
c_{i}=\alpha_{i} \boldsymbol{f}_{i}+\left(1-\alpha_{i}\right) \boldsymbol{b}_{i},
$$

where $\alpha i \in[0,1]$ represents the pixel's foreground opacity; $c i=[c 1 i \cdots c n i] \in$ $R^{n}$, the color vector; $f i=[f 1 i \cdots f n i] \in R^{n}$ and $b i=[b 1 i \cdots b n i] \in R^{n}$ represent the foreground and background color vectors at $i$ th pixel, respectively.

However, it is hard to generate a perfect matte from a given image without any prior information(e.g., which is given as a trimap) because the matting problem (2) is intrinsically ill-posed. In here, we take advantage of facial feature points to construct facial component templates which are equivalent to trimaps of each component (see Fig.2, Fig.4(b)). We also exploit each alpha matte obtained from digital matting later for assigning pseudo-depth values to the component subregions. Moreover, the 
obtained each eyeball is not complete image because some pixels in each eyeball are occluded by the eyelid. Therefore, we exploit a texture synthesis method [7] for restoring the corrupted partial view(see Fig.4(c)).

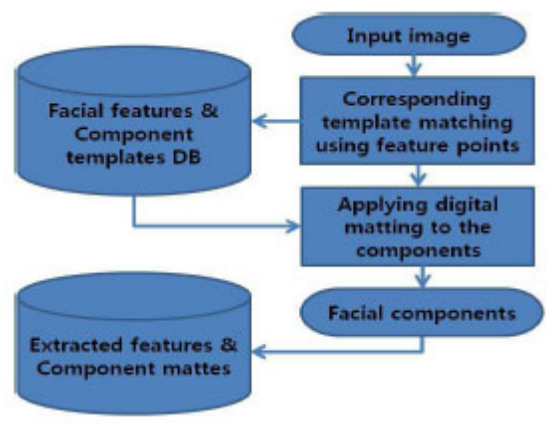

Fig. 2. Module of facial components extraction

\subsection{Facial Expression}

The changes like gaze and lip movements are very critical visual cues for realism of the facial expressions. We create realistic animation on a $2 \mathrm{D}$ face image by using conventional image warping method [8] as well as the obtained alpha mattes for representing pseudo-depth values. In order to express an independent movement of the lower lip, the zvalues of the lip are defined as follows:

$$
z_{m}=c *\left|\hat{y}-y_{m}\right|,
$$

where $z m$ and $y_{m}$ are coordinates of the lower lip. $c$ and $\hat{y}$ are a coefficient and jaw pivot point (see Fig.3), respectively. The lower lip region is separated from other regions by referencing the alpha values in their regions. That is, if specific alpha values are more than a defined threshold, their object would be regarded as a fore(e.g., lip) or background(e.g., eyeballs) region. Also, we formulate the lip opens with different angles $\gamma$ as follows:

$$
\left[\begin{array}{l}
\hat{z}^{\prime} \\
\hat{y}^{\prime}
\end{array}\right]=\left[\begin{array}{rr}
\cos \gamma & -\sin \gamma \\
\sin \gamma & \cos \gamma
\end{array}\right]\left[\begin{array}{l}
\hat{z} \\
\hat{y}
\end{array}\right],
$$

where $\hat{z}$ and $\hat{y}$ are coordinates of the closed lower lip, $\hat{z}^{\prime}$ and $\hat{y}^{\prime}$ are coordinate values when opening the lower lip. After projecting the translated coordinate values to the given image space, we just consider $\hat{x}$ and $\hat{y}^{\prime}$ values as a moving lip coordinates. Similarly, the movements of both eyeballs would be defined as the lips translation (4). In addition, we use a two-pass mesh warping method proposed by [8] for animating a variety of facial expressions. 


\section{Results}

We perform several experiments with real face image in order to validate our proposed scheme, and show some test results with various expressions. As shown in Fig.4 (a) and (b), the eyelids in the eyeball regions are not affected from the segmented both eyeballs. Such a delicate alpha matte of the eyeball makes it possible to animate in an efficient way more realistic facial appearances. Fig. 5 shows a variety of results generated from just one face input image of Fig.1. Any facial animation is synthesized as sequential movements of each facial component. Our system is developed in $\mathrm{C}++$ with openCV lib.
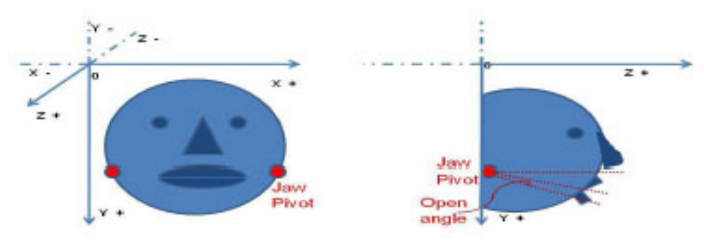

Fig. 3. Description of lip opens

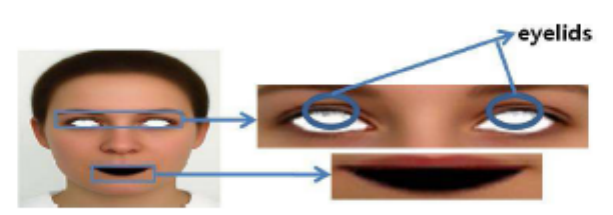

(a) Face image except the regions of eyeballs and lip.

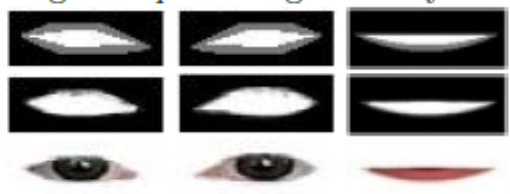

(b) Trimaps(top), alpha mattes(mid) and extracted components(bottom).

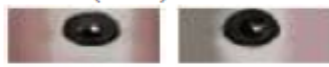

(c) Complete textures of both eyeballs.

Fig. 4. Results of components extraction \& reconstruction

\section{Conclusions and Further Works}

We have presented a realistic facial animation method with one two-dimensional face image. The major contribution of our proposed facial animation method could be summarized as follows: it shows how the facial animation with a variety of expressions can be generated realistically and easily, where main facial components extracted through the digital matting as well as pseudo-depth values inferred from the obtained alpha mattes are exploited in the system. Hence, there are some room for 


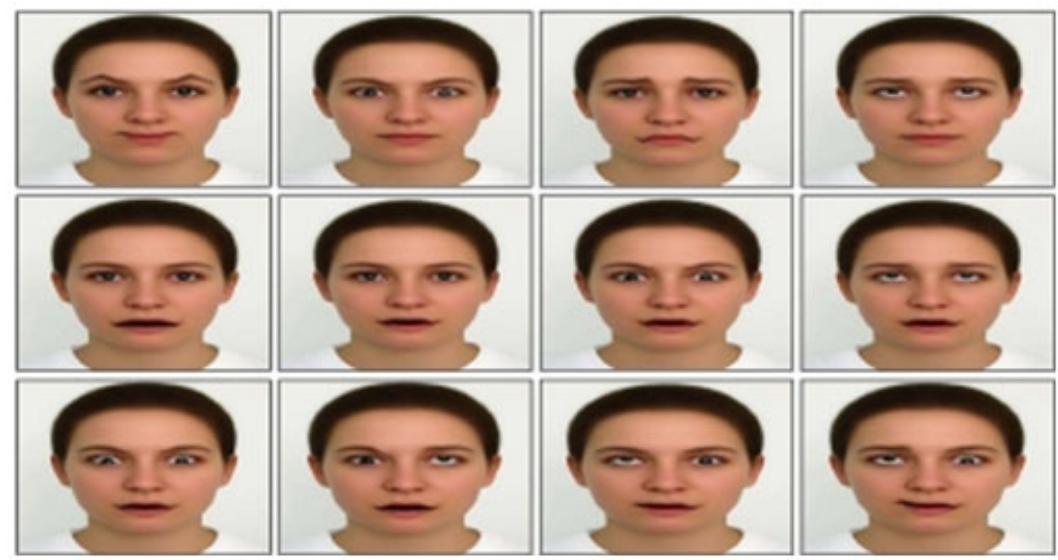

Fig. 5. Simulated results

improvement in our work. Currently the facial pose is limited to a frontal face. It is desirable to extend various facial poses for more realistic facial animations. Empirical results with real image confirmed that our method generates high quality frontal facial animation very efficiently.

\section{References}

1. Sifakis, E., Neverov, I., Fedkiw, R.: Automatic determination of facial muscle activations from sparse motion capture marker data. ACM Trans. Graphics 24(3), 417-425 (2005)

2. Deng, Z., Neumann, U.: Data-driven 3D facial animation. Springer, Heidelberg (2008)

3. Parke, F.I.: A parametric model for human faces, Ph.D. thesis, University of Utah (1974)

4. Parke, F.I.: Computer generated animation of faces. In: Proc. ACM National Conference, Boston, MA, pp. 451-457 (1974)

5. Parke, F.I., Waters, K.: Computer facial animation. A K Peters, Wellesley (1996)

6. Levin, A., Lischinski, D., Weiss, Y.: A closed form solution to natural image matting. IEEE Trans. Pattern Analysis and Machine Intelligence 30(2), 228-242 (2008)

7. Efros, A., Leung, T.: Texture synthesis by non-parametric sampling. In: Proc. Int'l Conf. Computer Vision, pp. 1033-1038 (2004)

8. Wolberg, G.: Digital image warping. IEEE Computer Society Press, Los Alamitos (1992)

9. Liu, Z., Shan, Y., Zhang, Z.: Expressive expression mapping with ratio images. In: Proc. SIGGRAPH Conf., pp. 271-276 (August 2001)

10. Song, M., Wang, H., Bu, J., Chen, C., Liu, Z.: Subtle facial expression modeling with vector field decomposition. In: Proc. Int'l Conf. Image Processing, pp. 2101-2104 (2006)

11. Bitouk, D., Nayar, S.K.: Creating a speech enabled avatar from a single photograph. In: Proc. IEEE Virtual Reality, pp. 107-110 (2008)

12. Wang, J., Cohen, M.: Image and Video Matting: A Survey. Foundations and Trends, vol. 3. Computer Graphics and Vision (2007)

13. Joachims, T.: Svm(support vector machine) light (2008) 